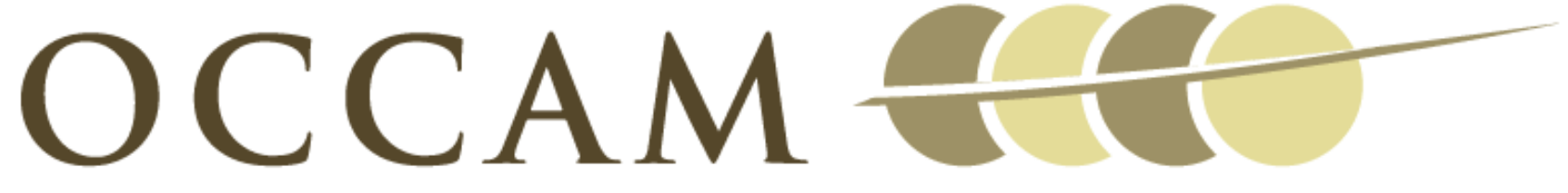

OXFORD CENTRE FOR COLLABORATIVE APPLIED MATHEMATICS

\author{
Report Number 11/64
}

Stability estimates for a twisted rod under terminal loads: a three-dimensional study

by

Apala Majumdar, Christopher Prior, and Alain Goriely

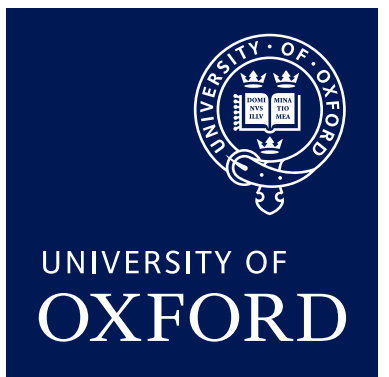

Oxford Centre for Collaborative Applied Mathematics Mathematical Institute 24 - 29 St Giles'

Oxford

OX1 3LB

England 



\title{
Stability estimates for a twisted rod under terminal loads: a three-dimensional study
}

\author{
Apala Majumdar · Christopher Prior · Alain Goriely
}

Received: date / Accepted: date

\begin{abstract}
The stability of an inextensible unshearable elastic rod with quadratic strain energy density subject to end loads is considered. We study the second variation of the corresponding rod-energy, making a distinction between in-plane and out-of-plane perturbations and isotropic and anisotropic cross-sections respectively. In all cases, we demonstrate that the naturally straight state is a local energy minimizer in parameter regimes specified by material constants. These stability results are also accompanied by instability results in parameter regimes defined in terms of material constants.
\end{abstract}

Keywords Cosserat rods $\cdot$ Stability of straight axis solution $\cdot$ Variational criterion

Mathematics Subject Classification (2000) 74B20 $\cdot 49 \mathrm{~K} 40$

\section{Introduction}

The buckling of rods under loads is the archetypical stability problem in continuum mechanics, dynamical systems, and bifurcation theory. First described by Euler for the case of a beam under compressive force [10,11], the general problem of stability of elastic structures under loads and constraints is a major topic in continuum mechanics of great technological importance [4]. There are essentially three methods to approach the problem of stability in rods. First, we have the method of static bifurcation as originally used by Michell [23,15] and Timoshenko [26]. This method consists in identifying values of a parameter (the control parameter) for which the linearised system (around a reference state) admits a non-trivial solution. This is accompanied by the assumption that the reference state of the system loses stability with respect to a bifurcating branch at the critical control parameter. This method can be easily applied to many systems with great success, leading to a simple and elegant way for developing some insight into possible behaviors of rods. However, it fails both in providing rigorous results (there is, in general, no basis for the assumed stability of the new branch) and in handling more complicated situations (for instance the case of a follower tangential load on a rod proposed by Beck [5] cannot be properly analyzed by a static bifurcation study). For these non-conservative problems, the dynamical bifurcation method is more appropriate. Within the framework of this second method, one studies the time evolution of small perturbations around both the trivial and bifurcated solutions for the linearized problem[28,1,17]. The spectrum of the linearized operator provides information about the exponential instability of some of these solutions which, for some prescribed dynamics, can yield rigorous results [6]. The third approach is a variational approach whereby we describe stable equilibria as local minimizers of a suitably defined elastic energy [7]. There are necessary and sufficient criteria for stability in terms of the second variation of the elastic energy [21] e.g. Jacobi's condition, Legendre's condition and these have been successfully applied to a large class of nonlinear rods [22].

While it is usually the trend to generalise methods and systems, we run the risk of not seeing the tree for the forest. Here, we present a self-contained proof for the stability of a naturally straight, inextensible, unshearable rod in the

Majumdar A, Prior C, Goriely A

OCCAM, Mathematical Institute, Oxford University, 24 - 29 St Giles', Oxford, England, OX1 3LB

+44(0) 1865615169

E-mail: goriely@maths.ox.ac.uk 
(a)

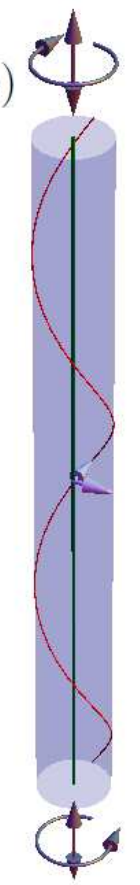

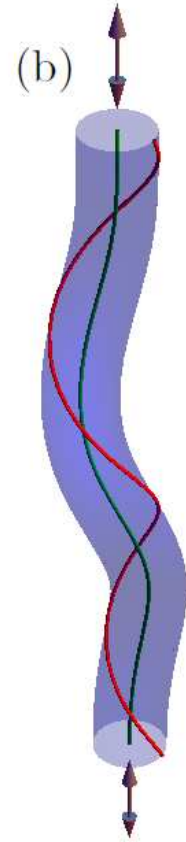

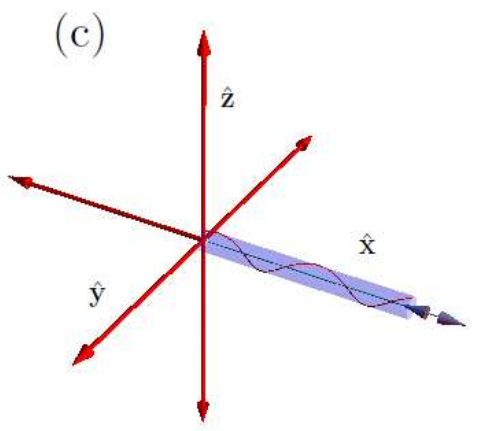

Fig. 1 A depiction of the type of rod geometry considered. The equilibrium configuration is shown in (a). The applied loads $F \hat{\mathbf{x}}$ are represented by bi-directional arrows, to indicate tension or compression. A moment pair used to apply a left-handed end-rotation $M$ is also shown. A curve drawn out by the vector $\mathbf{d}_{1}(s)$ is shown on the surface, indicating the uniform twist of the equilibrium solution we study. An example of the type of perturbation considered is shown in (b). The tangent vector $\mathbf{d}_{3}$ points along $\hat{\mathbf{x}}$ at the end points and the force is considered to act at the rod's ends along $\hat{\mathbf{x}}$. The center-line of the equilibrium configuration is aligned with the $\hat{\mathbf{x}}$ axis, as shown in (c).

$(x, y)$-plane, with a quadratic strain-energy density, under tension and controlled end-rotation. This problem is of great interest in the study of single-molecule DNA experiments [14]. As a stability problem, similar systems were first studied by Greenhill [18] and revisited by many authors through the method of static bifurcation or linear stability analysis [28]. We address the question of stability by studying the second variation of the strain energy directly i.e. without appealing to either Legendre or Jacobi conditions or conjugate point methods. We formulate the stability problem in terms of Euler angles $(\theta, \phi, \psi)$ (similar examples can be found in [21]) and by means of a direct computation, show that the energy density is strictly convex with respect to the first derivatives provided that the polar singularities, $\theta=0$ and $\theta=\pi$, are not encountered. The strict convexity allows us to infer the local energy minimality of the naturally straight state from the positivity of the second variation $[3,19]$. We use integral inequalities e.g. Wirtinger's inequality, to obtain explicit upper and lower bounds for the critical tension $F_{c}$ in terms of the material parameters, rod-length and end-rotation such that the rod is naturally straight for $F \geq F_{c}$ and deforms for $F<F_{c}$. For the case of a rod with an isotropic cross-section and subject to in-plane perturbations, these bounds match and yield an exact formula for the critical tension, which is simply the classical buckling result [25]. When we consider the fully three-dimensional problem with out-of-plane perturbations, these bounds do not match as they also depend on the magnitude of the controlled end-rotation, referred to as twist in the rest of the paper. We show that these methods can be readily generalized to rods with anisotropic cross-sections and the picture is qualitatively unchanged. The paper is organized as follows. We recall the main constituents of the elastic model in Section 2. In Section 3, we derive the governing equilibrium Euler-Lagrange equations and in Sections 4.1 and 4.2, we analyze the stability of a naturally straight rod with an anisotropic and isotropic cross-section respectively, in terms of the second variation of the strain energy. We summarize our main conclusions in Section 5.

\section{Preliminaries}

Consider a naturally straight, uniform, inextensible and unshearable elastic rod. We work with a Kirchhoff rod under the assumptions of hyper-elasticity (the existence of a strain energy density) and linear constitutive relations between moments and Darboux curvature $[20,21,2]$. 


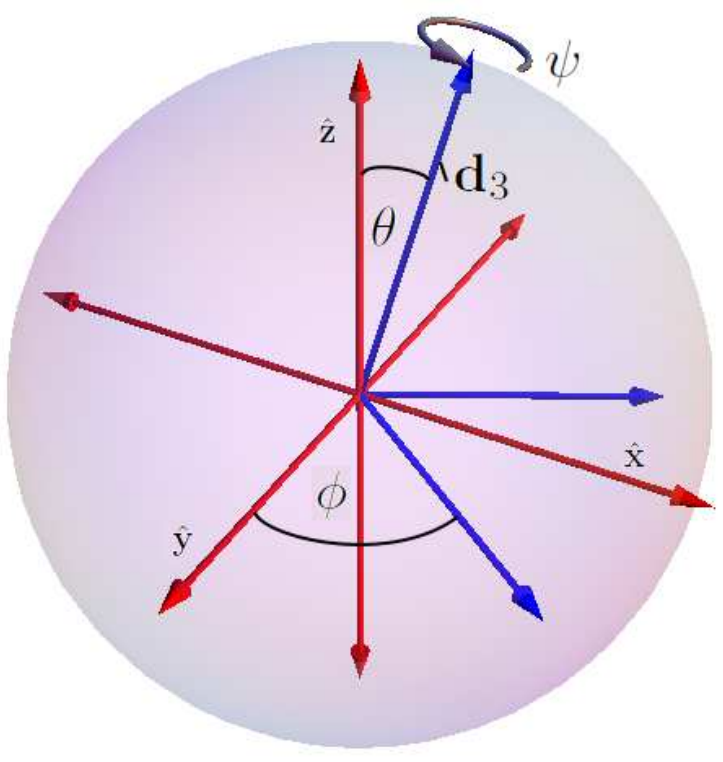

Fig. 2 A visualisation of the Euler angle rotations $\{\theta, \phi, \psi\}$, used to map the Cartesian basis $\{\hat{\mathbf{x}}, \hat{\mathbf{y}}, \hat{\mathbf{z}}\}$ to the director basis $\left\{\mathbf{d}_{1}, \mathbf{d}_{2}, \mathbf{d}_{3}\right\}$. First the Cartesian basis is rotated through an angle $\phi$ about $\hat{\mathbf{z}}$. A rotation of angle $\theta$ about the image of the $\hat{\mathbf{y}}$ vector is then applied. This maps the vector $\hat{\mathbf{z}}$ to $\mathbf{d}_{3}$. Finally a rotation of angle $\psi$ is applied about $\mathbf{d}_{3}$ in order to obtain the director basis.

The elastic rod is initially aligned along the $\hat{\mathbf{x}}$ axis of a Cartesian basis $\{\hat{\mathbf{x}}, \hat{\mathbf{y}}, \hat{\mathbf{z}}\}$. The rod is of fixed length $L$, is clamped at both ends and subject to a controlled end-rotation characterized by a non-zero parameter $M$, referred to as the twist parameter in the paper. An external force $F$, in the $x$-direction, is applied to both ends (see Figures 1 (a) and $1(\mathrm{c})$ ). We parameterize the rod by an arc-length variable, $s$, where $s \in[0, L]$. The state of the rod is specified by its center-line, $\mathbf{r}(s):[0, L] \rightarrow \mathbb{R}^{3}$, and a triad of orthogonal vectors known as directors: $\left\{\mathbf{d}_{1}, \mathbf{d}_{2}, \mathbf{d}_{3}\right\}$ where $\mathbf{d}_{3}=\frac{d \mathbf{r}}{d s}$ is the local tangent to the rod [21]. Collectively, the directors define a right-handed rod-centered co-ordinate frame that relates the stressed and unstressed states of the rod. The director evolution along the rod is governed by the following system of ordinary differential equations:

$$
\mathbf{d}_{i}^{\prime}=\mathbf{U} \times \mathbf{d}_{i} \quad i=1,2,3
$$

where $\mathbf{U}=\left(u_{1}, u_{2}, u_{3}\right)$ is the Darboux vector, $u_{1}$ and $u_{2}$ are related to the curvature and geometric torsion of the rod-axis and $u_{3}$ measures physical twist [21] (see Figures 1(a) and 1(b)). We note that $\mathbf{d}_{i}^{\prime}$ denotes the derivative of $\mathbf{d}_{i}$ with respect to $s$ and this notation will be followed throughout the paper.

We close the system by introducing a strain energy density function $W\left(u_{1}, u_{2}, u_{3}\right)$ that is quadratic in the strain components $[8,9]$,

$$
W\left(u_{1}, u_{2}, u_{3}\right)=\frac{1}{2} \mathbf{U}_{i} \mathbf{M}_{i j} \mathbf{U}_{j} \quad i, j=1,2,3,
$$

where we have used Einstein summation convention and $\mathbf{M} \in \mathbb{M}^{3 \times 3}$ is a constant, positive-definite symmetric $3 \times 3$ matrix whose components can be related to the elastic constants of the rod. In particular, the positive-definiteness requires that the diagonal elements, $\left\{M_{11}, M_{22}, M_{33}\right\}$, are positive where $M_{11}, M_{22}$ are the bending rigidities and $M_{33}$ is the torsional rigidity respectively [21].

In what follows, we introduce a set of Euler angles $\{\theta(s), \phi(s), \psi(s)\} \in C^{1}([0, L] ; \mathbb{R})$ ( $C^{1}$ denotes the space of continuously differentiable functions) to describe the orientation of the directors $\left\{\mathbf{d}_{i}(s)\right\}$ with respect to the unstressed state (see Figure 2). Following the conventions in [21], the strain components are given in terms of the Euler angles by

$$
\begin{aligned}
& u_{1}=-\phi^{\prime} \sin \theta \cos \psi+\theta^{\prime} \sin \psi, \\
& u_{2}=\phi^{\prime} \sin \theta \sin \psi+\theta^{\prime} \cos \psi, \\
& u_{3}=\phi^{\prime} \cos \theta+\psi^{\prime} .
\end{aligned}
$$


The clamped boundary conditions translate into a Dirichlet boundary-value problem for $\theta(s), \phi(s):[0, L] \rightarrow \mathbb{R}$ given by

$$
\begin{aligned}
& \theta(0)=\theta(L)=\frac{\pi}{2}, \\
& \phi(0)=\phi(L)=0,
\end{aligned}
$$

and the controlled end-rotation at both ends is incorporated into the boundary condition

$$
\begin{aligned}
& \psi(0)=0, \\
& \psi(L)=2 \pi M,
\end{aligned}
$$

for some non-zero integer $M$. The rod-energy is the sum of the strain energy (see (2)) and the potential energy as shown below

$$
\mathcal{E}[\theta, \phi, \psi]=\int_{0}^{L} W\left(u_{1}, u_{2}, u_{3}\right)-F \sin \theta \cos \phi d s
$$

In (6), the strain components are related to the Euler angles as in (3) and $F$ can be positive (stretching) or negative (compressive). An equilibrium is defined to be a solution of the Euler-Lagrange equations associated with (6) and a stable equilibrium is a local energy-minimizer in the space of admissible variations.

The unstressed state of the rod corresponds to the naturally straight state $\Theta_{0}(s)=\left(\theta_{0}(s), \phi_{0}(s), \psi_{0}(s)\right)$ defined by

$$
\begin{aligned}
& \theta_{0}(s)=\frac{\pi}{2} \quad 0 \leq s \leq L, \\
& \phi_{0}(s)=0 \quad 0 \leq s \leq L .
\end{aligned}
$$

The corresponding profile for $\psi_{0}$ will be determined from the Euler-Lagrange equations in the next section. We note that the first two strain components, $u_{1}$ and $u_{2}$, identically vanish for $s \in[0, L]$. In the following sections, we consider two questions (i) for what choices of the matrix $\mathbf{M}$ in (2) is the unstressed state an equilibrium state and (ii) for such choices of $\mathbf{M}$, when is the unstressed state a stable equilibrium and can we predict the onset of instabilities?

\section{The Euler-Lagrange Equations}

The energy density in (6) can be explicitly written in terms of the $M_{i j}$ 's in (2) as shown below

$$
e[\theta, \phi, \psi]=\frac{1}{2}\left\{M_{11} u_{1}^{2}+M_{22} u_{2}^{2}+M_{33} u_{3}^{2}+2 M_{12} u_{1} u_{2}+2 M_{13} u_{1} u_{3}+2 M_{23} u_{2} u_{3}\right\}-F \sin \theta \cos \phi,
$$

where the $u_{i}$ 's are given in terms of the Euler angles in (3). All equilibria associated with the rod-energy (6) are solutions of the following bulk Euler-Lagrange equations on the open interval $0<s<L$ (see [12] for an exposition on the methods in the calculus of variations):

$$
\begin{aligned}
& \sin \theta\left[-M_{11} u_{1} \cos \psi+M_{22} u_{2} \sin \psi\right]+M_{33} u_{3} \cos \theta++M_{12} \sin \theta\left[u_{1} \sin \psi-u_{2} \cos \psi\right] \\
& +M_{13}\left[u_{1} \cos \theta-u_{3} \sin \theta \cos \psi\right]+M_{23}\left[u_{2} \cos \theta+u_{3} \sin \theta \sin \psi\right]=F \sin \theta \sin \phi \\
& \frac{d}{d s}\left[M_{33} u_{3}+M_{13} u_{1}+M_{23} u_{2}\right] \\
& =u_{1} u_{2}\left(M_{11}-M_{22}\right)+M_{12}\left(u_{2}^{2}-u_{1}^{2}\right)+\left(M_{13} u_{2}-M_{23} u_{1}\right) u_{3},
\end{aligned}
$$

and

$$
\begin{aligned}
& \frac{d}{d s}\left[M_{11} u_{1} \sin \psi+M_{22} u_{2} \cos \psi+M_{12}\left(u_{1} \cos \psi+u_{2} \sin \psi\right)+M_{13} u_{3} \sin \psi+M_{23} u_{3} \cos \psi\right]= \\
& =\phi^{\prime} \cos \theta\left[M_{22} u_{2} \sin \psi-M_{11} u_{1} \cos \psi\right]-M_{33} u_{3} \phi^{\prime} \sin \theta-F \cos \theta \cos \phi+ \\
& +M_{12} \phi^{\prime} \cos \theta\left(u_{1} \sin \psi-u_{2} \cos \psi\right)+M_{13} \phi^{\prime}\left(-u_{1} \sin \theta-u_{3} \cos \theta \cos \psi\right)+ \\
& +M_{23} \phi^{\prime}\left(-u_{2} \sin \theta+u_{3} \cos \theta \sin \psi\right) .
\end{aligned}
$$


We now address the question - for what choices of $\left\{M_{11}, M_{22}, M_{33}, M_{12}, M_{13}, M_{23}\right\}$ is the unstressed state defined in (7) an equilibrium state? We denote the Darboux vector associated with the unstressed state by $\mathbf{U}_{0}=$ $\left\{u_{10}, u_{20}, u_{30}\right\}$ with $u_{10}=u_{20}=0$. We substitute $u_{10}=u_{20}=0$ and $\theta_{0}(s)=\frac{\pi}{2}$ for $0 \leq s \leq L$ into (10) to find

$$
M_{33} u_{3}=\text { constant }
$$

i.e. the unstressed state has constant twist density $u_{3}$. Recalling that $\phi_{0}$ is a constant from (7) and the controlled end-rotation boundary conditions (5), we necessarily have that

$$
\psi_{0}^{\prime}(s)=\frac{2 \pi M}{L}
$$

and hence,

$$
\psi_{0}(s)=2 \pi M \frac{s}{L} \quad 0 \leq s \leq L
$$

However, (9) requires that

$$
M_{23} u_{3} \sin \psi_{0}-M_{13} u_{3} \cos \psi_{0}=0
$$

since $\phi_{0}=0$, and this is clearly incompatible with (13). We deduce that the off-diagonal elements

$$
M_{13}=M_{23}=0
$$

for the unstressed state $\Theta_{0}=\left(\theta_{0}, \phi_{0}, \psi_{0}\right)$ to be an admissible equilibrium. Physically the constraints (14) imply that there is no coupling between bending and torsion.

Comment: We note that for the rod-energy (6) to have any straight equilibrium state characterized by $\theta(s)=$ $C_{1}$ and $\phi(s)=C_{2}$, for constants $C_{1}$ and $C_{2}$, we must have $M_{13}=M_{23}=0$.

For a uniform rod with the director $\mathbf{d}_{3}$ aligned along the local tangent to the rod, we can WLOG take $M_{12}=0$ [8]. This combined with (14) shows that it suffices to consider the diagonal rod-energy

$$
\mathcal{E}_{D}[\theta, \phi, \psi]=\int_{0}^{L} \frac{1}{2}\left(A u_{1}^{2}+B u_{2}^{2}+C u_{3}^{2}\right)-F \sin \theta \cos \phi d s
$$

whilst studying the unstressed state $\Theta_{0}=\left(\theta_{0}, \phi_{0}, \psi_{0}\right)$ defined in (7) and (13) above. Here, $A, B, C>0$ are materialdependent constants. If $A \neq B$, then the rod has an anisotropic cross-section whereas if $A=B$, then the rod cross-section is isotropic [21]. We refer to the $A \neq B$ case as the anisotropic case and to the $A=B$ case as the isotropic case in what follows. In the anisotropic case, we can always assume that

$$
A \leq B
$$

and this convention will be followed in the rest of the paper [16].

For completeness, the Euler-Lagrange equations associated with the diagonal rod-energy in (15) are given below,

$$
\begin{aligned}
& \frac{d}{d s}\left[-A u_{1} \sin \theta \cos \psi+B u_{2} \sin \theta \sin \psi+C u_{3} \cos \theta\right]=F \sin \theta \sin \phi \\
& C \frac{d u_{3}}{d s}=u_{1} u_{2}(A-B) \\
& \frac{d}{d s}\left[A u_{1} \sin \psi+B u_{2} \cos \psi\right]= \\
& =\phi^{\prime} \cos \theta\left[B u_{2} \sin \psi-A u_{1} \cos \psi\right]-C u_{3} \phi^{\prime} \sin \theta-F \cos \theta \cos \phi .
\end{aligned}
$$

We note that the corresponding equilibria do not have, in general, constant twist density $u_{3}$ for $A \neq B$. 


\section{Stability Analysis}

\subsection{The Anisotropic Case}

In the previous section, we demonstrated that the unstressed state $\Theta_{0}=\left(\theta_{0}, \phi_{0}, \psi_{0}\right)$, in (7) and (13), is an equilibrium state for the diagonal rod-energy in (15) for all choices of $A, B, C>0$. In what follows, we take $M>0$ and treat (4) and (5) collectively as a Dirichlet boundary-value problem for the Euler angles $(\theta, \phi, \psi)$. We first make the elementary observation that the unstressed state is not globally energy-minimizing for large, compressive forces $F<0$ in $(15)$.

Lemma 1 For $F<0$ and $|F|$ sufficiently large, the planar equilibrium $\Theta_{0}(s)=\left(\theta_{0}, \phi_{0}, \psi_{0}\right)$ for $s \in[0, L]$, is not a global minimizer for the rod-energy in (15), subject to the boundary conditions (4) and (5).

Proof: Recalling that $\theta_{0}=\frac{\pi}{2}, \phi_{0}=0$ and $\psi_{0}(s)=2 \pi M \frac{s}{L}$, a direct computation shows that the rod-energy of the unstressed state is given by

$$
\mathcal{E}_{D}\left[\theta_{0}, \phi_{0}, \psi_{0}\right]=2 \pi^{2} C \frac{M^{2}}{L}+|F| L
$$

We construct a function $\theta^{*}:[0, L] \rightarrow \mathbb{R}$ subject to the clamped boundary conditions such that

$$
\mathcal{E}_{D}\left[\theta^{*}, \phi_{0}, \psi_{0}\right]<\mathcal{E}_{D}\left[\theta_{0}, \phi_{0}, \psi_{0}\right]
$$

for $|F|$ sufficiently large, thus proving the claim in Lemma 1 . The function $\theta^{*}$ is defined to be

$$
\theta^{*}(s)= \begin{cases}\frac{\pi}{2}\left(1-\frac{s}{L}\right) & 0 \leq s \leq \frac{L}{4} \\ \frac{3 \pi}{8} & \frac{L}{4} \leq s \leq \frac{3 L}{4} \\ \frac{\pi}{2} \frac{s}{L} & \frac{3 L}{4} \leq s \leq L\end{cases}
$$

Comment: $\theta^{*}$ in (22) is not continuously differentiable by construction. However, $\theta^{*}$ can be approximated by continuously differentiable functions and this will not affect the energy estimates below.

The rod-energy of the triplet $\Theta^{*}=\left(\theta^{*}, \phi_{0}, \psi_{0}\right)$ is bounded from above by -

$$
\begin{aligned}
& \mathcal{E}_{D}\left[\theta^{*}, \phi_{0}, \psi_{0}\right] \leq \frac{B}{2} \int_{0}^{L}\left(\frac{d \phi_{0}}{d s}\right)^{2} \sin ^{2} \theta^{*}(s)+\left(\frac{d \theta^{*}}{d s}\right)^{2} d s+ \\
& +\int_{0}^{L} \frac{C}{2}\left(\frac{d \phi_{0}}{d s} \cos \theta^{*}(s)+\frac{d \psi_{0}}{d s}\right)^{2}+|F| \sin \theta^{*}(s) d s
\end{aligned}
$$

since $A \leq B$ from (16). Since $\phi_{0}(s)=0$ and $\psi_{0}(s)=2 \pi M \frac{s}{L}$ for $s \in[0, L]$, we have that

$$
\frac{C}{2} \int_{0}^{L}\left(\frac{d \phi_{0}}{d s} \cos \theta^{*}(s)+\frac{d \psi_{0}}{d s}\right)^{2} d s=2 \pi^{2} M^{2} \frac{C}{L} .
$$

From the explicit form of $\theta^{*}$ in $(22)$, we have

$$
\begin{aligned}
& \int_{0}^{L}\left(\frac{d \theta^{*}}{d s}\right)^{2} d s=\frac{\pi^{2}}{8 L} \text { and } \\
& \int_{0}^{L}|F| \sin \theta^{*}(s) d s \leq|F| \frac{L}{2}+\frac{|F| L}{2} \sin \frac{3 \pi}{8} .
\end{aligned}
$$

Substituting the above into (23), we have that

$$
\mathcal{E}_{D}\left[\theta^{*}, \phi_{0}, \psi_{0}\right] \leq \frac{B \pi^{2}}{16 L}+2 \pi^{2} M^{2} \frac{C}{L}+|F| \frac{L}{2}\left(1+\sin \frac{3 \pi}{8}\right) .
$$

We deduce that (21) will be satisfied whenever

$$
\frac{B \pi^{2}}{16 L} \leq|F| L\left(1-\frac{1}{2}\left(1+\sin \frac{3 \pi}{8}\right)\right) .
$$


This inequality is satisfied for large $|F|$ since $\left(1-\frac{1}{2}\left(1+\sin \frac{3 \pi}{8}\right)\right)>0$, hence proving the claim in Lemma 1 .

The next step is to address the question of stability of the unstressed state and study the interplay between the elastic constants, twist, rod-length, and external force. To this end, we first recall a version of Weierstrass' theorem for one-dimensional problems $[3,19,21]$ : consider the integral $I(y)=\int_{a}^{b} f\left(x, y(x), y^{\prime}(x)\right) d x$ where $y^{\prime}(x)=\frac{d y}{d x}$, subject to Dirichlet boundary conditions at the end-points $x=a$ and $x=b$. The Weierstrass theory guarantees that if $f$ is smooth in its arguments, $f_{y^{\prime} y^{\prime}}>0$ i.e. $f$ is strictly convex with respect to the gradient, then an equilibrium solution $u \in C^{2}[a, b]$ is a local energy minimizer if the second variation $\delta^{2} I[u]>0$. We can explicitly compute the $3 \times 3$ matrix, $e[\theta, \phi, \psi]_{y^{\prime} y^{\prime}}$, where $y^{\prime}=\left(\theta^{\prime}, \phi^{\prime}, \psi^{\prime}\right)$ and $e[\theta, \phi, \psi]$ has been defined in (8). For completeness, the matrix $e[\theta, \phi, \psi]_{y^{\prime} y^{\prime}}$ is given by

$$
e[\theta, \phi, \psi]_{y^{\prime} y^{\prime}}=\left(\begin{array}{ccc}
\frac{d^{2} e}{d \theta^{\prime 2}} & \frac{d^{2} e}{d \theta^{\prime} d \phi^{\prime}} & \frac{d^{2} e}{d \theta^{\prime} d \psi^{\prime}} \\
\frac{d^{2} e}{d \theta^{\prime} d \phi^{\prime}} & \frac{d^{2} e}{d \phi^{\prime 2}} & \frac{d^{2} e}{d \psi^{\prime} d \phi^{\prime}} \\
\frac{d^{2} e}{d \theta^{\prime} d \psi^{\prime}} & \frac{d^{2} e}{d \psi^{\prime} d \phi^{\prime}} & \frac{d^{2} e}{d \psi^{\prime 2}}
\end{array}\right)
$$

For the unstressed state $\Theta_{0}$ defined in (7) and (13), this matrix is given by

$$
\mathbf{T}\left(\Theta_{0}\right)=\left(\begin{array}{ccc}
A \sin ^{2} \psi_{0}+B \cos ^{2} \psi_{0} & (B-A) \sin \psi_{0} \cos \psi_{0} & 0 \\
(B-A) \sin \psi_{0} \cos \psi_{0} & A \cos ^{2} \psi_{0}+B \sin ^{2} \psi_{0} & 0 \\
0 & 0 & C
\end{array}\right)
$$

and one can directly check that

$$
\mathbf{h}_{i} \mathbf{T}\left(\Theta_{0}\right)_{i j} \mathbf{h}_{j}=B\left(\cos \psi_{0} h_{1}+\sin \psi_{0} h_{2}\right)^{2}+A\left(\sin \psi_{0} h_{1}-\cos \psi_{0} h_{2}\right)^{2}+C h_{3}^{2}>0
$$

for any non-zero vector $\mathbf{h}=\left(h_{1}, h_{2}, h_{3}\right) \in \mathbb{R}^{3}$. This necessarily implies that the eigenvalues of the matrix, $\mathbf{T}\left(\Theta_{0}\right)$, are postive. Hence, the energy density in (8) is strictly convex with respect to the first derivatives of the Euler angles in a neighbourhood of the planar equilibrium $\Theta_{0}$ and we can treat the positivity of the second variation as a sufficient criterion for local energy minimality [19].

For the sake of illustration, we repeat the same computation for the polar singularity $\theta=0$ i.e. compute the $3 \times 3$ matrix, $e[\theta, \phi, \psi]_{y^{\prime} y^{\prime}}$, where $y^{\prime}=\left(\theta^{\prime}, \phi^{\prime}, \psi^{\prime}\right)$, with $\theta=0$. The corresponding matrix is given by

$$
\mathbf{T}(0)=\left(\begin{array}{cccc}
A \sin ^{2} \psi+B \cos ^{2} \psi & 0 & 0 \\
0 & & C & C \\
0 & & C & C
\end{array}\right)
$$

so that

$$
\mathbf{h}_{i} \mathbf{T}(0)_{i j} \mathbf{h}_{j}=\left(A \sin ^{2} \psi+B \cos ^{2} \psi\right) h_{1}^{2}+C\left(h_{2}+h_{3}\right)^{2}
$$

for any non-zero vector $\mathbf{h}=\left(h_{1}, h_{2}, h_{3}\right) \in \mathbb{R}^{3}$. If $\mathbf{h} \in \mathbb{R}^{3}$ is such that $h_{1}=0$ and $h_{2}+h_{3}=0$, then the right-hand side of the above equality vanishes and hence, we lose strict convexity of the energy density with respect to the first derivatives near a polar singularity.

We non-dimensionalize the diagonal rod-energy in (15) by using the simple change of variable

$$
\bar{s}=\frac{s}{L} \quad 0 \leq \bar{s} \leq 1
$$

Then the re-normalized rod-energy is given by (up to a multiplicative constant)

$$
\mathcal{E}_{D}[\theta, \phi, \psi]=\int_{0}^{1} \frac{1}{2}\left(A u_{1}^{2}+B u_{2}^{2}+C u_{3}^{2}\right)-F L^{2} \sin \theta \cos \phi d \bar{s}
$$

In what follows, we work with the re-normalized energy in (28) and drop the bars from the re-scaled variable for brevity. All subsequent results are to be understood in terms of the re-scaled arc-length variable above. The unstressed state is now defined by the triplet

$$
\Theta_{0}(s)=\left(\theta_{0}, \phi_{0}, \psi_{0}\right)=\left(\frac{\pi}{2}, 0,2 \pi M s\right)
$$


We consider in-plane perturbations (perturbations in the (xy)-plane) and out-of-plane perturbations (three-dimensional perturbations) separately. To this end, for a given $M>0$, the general form of the in-plane perturbations of the unstressed state are given by $\Theta_{\epsilon}=\left(\theta_{\epsilon}, \phi_{\epsilon}, \psi_{\epsilon}\right)$ where

$$
\begin{aligned}
& \theta_{\epsilon}(s)=\frac{\pi}{2} \quad 0 \leq s \leq L, \\
& \phi_{\epsilon}(s)=\epsilon \phi_{1}(s), \\
& \psi_{\epsilon}(s)=2 \pi M s+\epsilon \psi_{1}(s),
\end{aligned}
$$

and $\phi_{1}(0)=\phi_{1}(1)=0$ and $\psi_{1}(0)=\psi_{1}(1)=0$ by virtue of the Dirichlet conditions in (4) and (5). The out-of-plane perturbations are described by perturbations in the Euler angle $\theta$ and the general form $\Theta_{\epsilon}=\left(\theta_{\epsilon}, \phi_{\epsilon}, \psi_{\epsilon}\right)$ is defined to be

$$
\begin{aligned}
& \theta_{\epsilon}(s)=\frac{\pi}{2}+\epsilon \theta_{1}(s), \\
& \phi_{\epsilon}(s)=\epsilon \phi_{1}(s), \\
& \psi_{\epsilon}(s)=2 \pi M s+\epsilon \psi_{1}(s),
\end{aligned}
$$

where $\theta_{1}(0)=\theta_{1}(1)=0, \phi_{1}(0)=\phi_{1}(1)=0$ and $\psi_{1}(0)=\psi_{1}(1)=0$ from the clamped boundary conditions (4) and the controlled end-rotation (5). We note that $\epsilon$ is sufficiently small so that $\theta_{\epsilon} \in(0, \pi)$ i.e. $\theta_{\epsilon}$ is bounded away from the polar singularities. From standard results in the calculus of variations [19,25], the positivity of the second variation of the rod-energy

$$
\left.\frac{d^{2}}{d \epsilon^{2}} \mathcal{E}_{D}\left(\theta_{\epsilon}, \phi_{\epsilon}, \psi_{\epsilon}\right)\right|_{\epsilon=0}>0
$$

is sufficient to guarantee the local energy minimality of the unstressed state. Similarly, if we can construct an explicit perturbation $\Theta_{\epsilon}=\left(\theta_{\epsilon}, \phi_{\epsilon}, \phi_{\epsilon}\right)$ for which

$$
\left.\frac{d^{2}}{d \epsilon^{2}} \mathcal{E}_{D}\left(\theta_{\epsilon}, \phi_{\epsilon}, \psi_{\epsilon}\right)\right|_{\epsilon=0}<0,
$$

then this is sufficient to deduce the instability of the unstressed state for the corresponding parameter regime.

Our first result concerns in-plane perturbations of the unstressed state where we recover the classical buckling result.

Proposition 1 The unstressed state defined in (7) and (13) is stable with respect to in-plane perturbations of the form (30) if

$$
F+\frac{A \pi^{2}}{L^{2}} \geq 0
$$

where $A$ is the smaller of the two bending stiffnesses $\{A, B\}$ from (16).

Proof: Consider the perturbations defined in (30); these are the most general in-plane perturbations compatible with this boundary-value problem. We denote the corresponding perturbed Darboux vector by $\mathbf{U}_{\epsilon}=\left(u_{1 \epsilon}, u_{2 \epsilon}, u_{3 \epsilon}\right)$ with strain components given by

$$
\begin{aligned}
& u_{1 \epsilon}=-\epsilon \phi_{1}^{\prime} \cos \psi_{0}, \\
& u_{2 \epsilon}=\epsilon \phi_{1}^{\prime} \sin \psi_{0}, \\
& u_{3 \epsilon}=2 \pi M+\epsilon \psi_{1}^{\prime} .
\end{aligned}
$$

We compute the corresponding second variation of the diagonal rod-energy in (15) to be

$$
\left.\frac{d^{2}}{d \epsilon^{2}} \mathcal{E}_{D}\left(\theta_{\epsilon}, \phi_{\epsilon}, \psi_{\epsilon}\right)\right|_{\epsilon=0}=\int_{0}^{1} A\left(\frac{d \phi_{1}}{d s}\right)^{2} \cos ^{2} \psi_{0}+B\left(\frac{d \phi_{1}}{d s}\right)^{2} \sin ^{2} \psi_{0}+C\left(\frac{d \psi_{1}}{d s}\right)^{2}+F L^{2} \phi_{1}^{2} d s
$$

The integral in (36) is bounded from below by

$$
\left.\frac{d^{2}}{d \epsilon^{2}} \mathcal{E}_{D}\left(\theta_{\epsilon}, \phi_{\epsilon}, \psi_{\epsilon}\right)\right|_{\epsilon=0} \geq \int_{0}^{1} A\left(\frac{d \phi_{1}}{d s}\right)^{2}+C\left(\frac{d \psi_{1}}{d s}\right)^{2}+F L^{2} \phi_{1}^{2} d s
$$

since $A \leq B$ from (16). Recalling Wirtinger's inequality [27]

$$
\int_{0}^{1}\left(\frac{d \phi_{1}}{d s}\right)^{2} \geq \pi^{2} \int_{0}^{1} \phi_{1}^{2}(s) d s
$$


we deduce that

$$
\left.\frac{d^{2}}{d \epsilon^{2}} \mathcal{E}_{D}\left(\theta_{\epsilon}, \phi_{\epsilon}, \psi_{\epsilon}\right)\right|_{\epsilon=0} \geq \int_{0}^{1}\left(A+F L^{2}\right) \phi_{1}^{2} d s
$$

and the second variation in (38) is clearly positive if $A+F L^{2}>0$ as stated in Proposition 1.

The borderline case $F=-A \frac{\pi^{2}}{L^{2}}$ : for the general in-plane perturbations in (30), one can perform a Taylor series expansion for the energy of the triplet $\left(\theta_{\epsilon}, \phi_{\epsilon}, \psi_{\epsilon}\right)$ as shown below:

$$
\begin{aligned}
& \mathcal{E}_{D}\left(\theta_{\epsilon}, \phi_{\epsilon}, \psi_{\epsilon}\right)=\mathcal{E}_{D}\left(\theta_{0}, \phi_{0}, \psi_{0}\right)+\left.\epsilon \frac{d \mathcal{E}_{D}\left(\theta_{\epsilon}, \phi_{\epsilon}, \psi_{\epsilon}\right)}{d \epsilon}\right|_{\epsilon=0}+\left.\frac{\epsilon^{2}}{2} \frac{d^{2}}{d \epsilon^{2}} \mathcal{E}_{D}\left(\theta_{\epsilon}, \phi_{\epsilon}, \psi_{\epsilon}\right)\right|_{\epsilon=0}+ \\
& +\left.\frac{\epsilon^{3}}{6} \frac{d^{3}}{d \epsilon^{3}} \mathcal{E}_{D}\left(\theta_{\epsilon}, \phi_{\epsilon}, \psi_{\epsilon}\right)\right|_{\epsilon=0}+\left.\frac{\epsilon^{4}}{24} \frac{d^{4}}{d \epsilon^{4}} \mathcal{E}_{D}\left(\theta_{\epsilon}, \phi_{\epsilon}, \psi_{\epsilon}\right)\right|_{\epsilon=0}+0\left(\epsilon^{5}\right) .
\end{aligned}
$$

The first variation vanishes since $\left(\theta_{0}, \phi_{0}, \psi_{0}\right)$ is an equilibrium solution. The second variation is non-negative from (38). The third variation vanishes, as can be checked from the formula for the perturbed strain vector for in-plane perturbations from (35). Finally

$$
\left.\frac{d^{4}}{d \epsilon^{4}} \mathcal{E}_{D}\left(\theta_{\epsilon}, \phi_{\epsilon}, \psi_{\epsilon}\right)\right|_{\epsilon=0}=-F L^{2} \int_{0}^{1} \phi_{1}^{4}(s) d s>0
$$

since $F=-A \frac{\pi^{2}}{L^{2}}<0$. We conclude that the unstressed state is stable with respect to in-plane perturbations for $F \geq-A \frac{\pi^{2}}{L^{2}}$.

We, next, address the question of stability with respect to out-of-plane perturbations.

Proposition 2 The unstressed state, defined in (7) and (13), is a local minimizer of the rod-energy (15) (is nonlinearly stable) under the following conditions

$$
\begin{aligned}
& A \geq 2 M C \\
& F>-A \frac{\pi^{2}}{L^{2}}+2 \pi M \pi^{2} \frac{C}{L^{2}} \text { and } \\
& F>-A \frac{\pi^{2}}{L^{2}}+\frac{2 \pi M C}{L^{2}}
\end{aligned}
$$

where $A$ is the smaller of the two bending stiffnesses $\{A, B\}$ from (16).

Proof: Consider the perturbations defined in (31); these are the most general three-dimensional perturbations compatible with this boundary-value problem. We denote the corresponding perturbed Darboux vector by $\mathbf{U}_{\epsilon}=\left(u_{1 \epsilon}, u_{2 \epsilon}, u_{3 \epsilon}\right)$ with strain components given by

$$
\begin{aligned}
& u_{1 \epsilon}=\epsilon\left(-\phi_{1}^{\prime} \cos (2 \pi M s)+\theta_{1}^{\prime} \sin (2 \pi M s)\right) \\
& u_{2 \epsilon}=\epsilon\left(\phi_{1}^{\prime} \sin (2 \pi M s)+\theta_{1}^{\prime} \cos (2 \pi M s)\right) \\
& u_{3 \epsilon}=2 \pi M+\epsilon \psi_{1}^{\prime}-\epsilon^{2} \theta_{1} \phi_{1}^{\prime} .
\end{aligned}
$$

The second variation of the rod-energy is computed to be

$$
\begin{aligned}
& \left.\frac{d^{2}}{d \epsilon^{2}} \mathcal{E}_{D}\left(\theta_{\epsilon}, \phi_{\epsilon}, \psi_{\epsilon}\right)\right|_{\epsilon=0}=\int_{0}^{1} A\left(-\phi_{1}^{\prime} \cos (2 \pi M s)+\theta_{1}^{\prime} \sin (2 \pi M s)\right)^{2} d s+ \\
& +\int_{0}^{1} B\left(\phi_{1}^{\prime} \sin (2 \pi M s)+\theta_{1}^{\prime} \cos (2 \pi M s)\right)^{2} d s+ \\
& +\int_{0}^{1} C\left(\psi_{1}^{\prime}\right)^{2}-4 \pi M C \phi_{1}^{\prime} \theta_{1}+F\left(\theta_{1}^{2}+\phi_{1}^{2}\right) d s
\end{aligned}
$$

and is bounded from below by

$$
\begin{aligned}
& \left.\frac{d^{2}}{d \epsilon^{2}} \mathcal{E}_{D}\left(\theta_{\epsilon}, \phi_{\epsilon}, \psi_{\epsilon}\right)\right|_{\epsilon=0} \geq A \int_{0}^{1}\left(\frac{d \theta_{1}}{d s}\right)^{2}+\left(\frac{d \phi_{1}}{d s}\right)^{2} d s+ \\
& +\int_{0}^{1} C\left(\frac{d \psi_{1}}{d s}\right)^{2}-4 \pi M C \theta_{1} \frac{d \phi_{1}}{d s}+F L^{2}\left(\theta_{1}^{2}+\phi_{1}^{2}\right) d s
\end{aligned}
$$


We partition the integral in (43) as shown below

$$
\begin{aligned}
& \left.\frac{d^{2}}{d \epsilon^{2}} \mathcal{E}_{D}\left(\theta_{\epsilon}, \phi_{\epsilon}, \psi_{\epsilon}\right)\right|_{\epsilon=0} \geq 2 \pi M C \int_{0}^{1}\left(\frac{d \phi_{1}}{d s}-\theta_{1}\right)^{2} d s+C \int_{0}^{1}\left(\frac{d \psi_{1}}{d s}\right)^{2} d s+ \\
& +\int_{0}^{1}(A-2 \pi M C)\left(\frac{d \phi_{1}}{d s}\right)^{2}+F L^{2} \phi_{1}^{2} d s+ \\
& +\int_{0}^{1} A\left(\frac{d \theta_{1}}{d s}\right)^{2}+\left(F L^{2}-2 \pi M C\right) \theta_{1}^{2} d s .
\end{aligned}
$$

The integral $\int_{0}^{1} 2 \pi M C\left(\frac{d \phi_{1}}{d s}-\theta_{1}\right)^{2} d s+C \int_{0}^{1}\left(\frac{d \psi_{1}}{d s}\right)^{2} d s$ is non-negative since $M, C>0$. If $A \geq 2 \pi M C$, then we can apply Wirtinger's inequality to the $\phi$-integral in (44) to get the following lower bound

$$
\int_{0}^{1}(A-2 \pi M C)\left(\frac{d \phi_{1}}{d s}\right)^{2}+F L^{2} \phi_{1}^{2} d s \geq \int_{0}^{1}\left[(A-2 \pi M C) \pi^{2}+F L^{2}\right] \phi_{1}^{2} d s
$$

and the integral in question is necessarily positive for a non-zero $\phi_{1}$ if

$$
\left[(A-2 \pi M C) \pi^{2}+F L^{2}\right]>0 .
$$

Similarly, we can obtain a lower bound for the $\theta$-integral in (44),

$$
\int_{0}^{1} A\left(\frac{d \theta_{1}}{d s}\right)^{2}+\left(F L^{2}-2 \pi M C\right) \theta_{1}^{2} d s \geq \int_{0}^{1}\left[A \pi^{2}+\left(F L^{2}-2 \pi M C\right)\right] \theta_{1}^{2} d s
$$

and this integral is necessarily positive for a non-zero $\theta_{1}$ if

$$
\left[A \pi^{2}+\left(F L^{2}-2 \pi M C\right)\right]>0 .
$$

We combine (45) and (46) to deduce that the unstressed state is a local minimizer of the rod-energy in (28) if

$$
\min \left\{A-2 \pi M C, A \frac{\pi^{2}}{L^{2}}+F-2 \pi M \frac{C}{L^{2}},(A-2 \pi M C) \frac{\pi^{2}}{L^{2}}+F\right\}>0 .
$$

The conclusion of Proposition 2 now follows.

Proposition 3 The unstressed state defined in (7) and (13) is unstable if

$$
F<-B \frac{\pi^{2}}{L^{2}}
$$

where $B$ is the larger of the two bending stiffnesses from (16).

Proof: We start with the integral formula for the second variation in (42) and make the elementary observation that

$$
\begin{aligned}
& \left.\frac{d^{2}}{d \epsilon^{2}} \mathcal{E}_{D}\left(\theta_{\epsilon}, \phi_{\epsilon}, \psi_{\epsilon}\right)\right|_{\epsilon=0} \leq \int_{0}^{1} B\left[\left(\frac{d \phi_{1}}{d s}\right)^{2}+\left(\frac{d \theta_{1}}{d s}\right)^{2}\right] d s+ \\
& +\int_{0}^{1} C\left(\frac{d \psi_{1}}{d s}\right)^{2}-4 \pi M C \theta_{1} \frac{d \phi_{1}}{d s}+F L^{2}\left(\theta_{1}^{2}+\phi_{1}^{2}\right) d s
\end{aligned}
$$

since $A \leq B$ from (16). We partition the integral in (49) as in (44) :

$$
\begin{aligned}
& \left.\frac{d^{2}}{d \epsilon^{2}} \mathcal{E}_{D}\left(\theta_{\epsilon}, \phi_{\epsilon}, \psi_{\epsilon}\right)\right|_{\epsilon=0} \leq 2 \pi M C \int_{0}^{1}\left(\frac{d \phi_{1}}{d s}-\theta_{1}\right)^{2} d s+C \int_{0}^{1}\left(\frac{d \psi_{1}}{d s}\right)^{2} d s+ \\
& +\int_{0}^{1}(B-2 \pi M C)\left(\frac{d \phi_{1}}{d s}\right)^{2}+F L^{2} \phi_{1}^{2} d s+ \\
& +\int_{0}^{1} B\left(\frac{d \theta_{1}}{d s}\right)^{2}+\left(F L^{2}-2 \pi M C\right) \theta_{1}^{2} d s .
\end{aligned}
$$


We aim to construct a triplet $\left(\theta_{1}, \phi_{1}, \psi_{1}\right)$ for which $\left.\frac{d^{2}}{d \epsilon^{2}} \mathcal{E}_{D}\left(\theta_{\epsilon}, \phi_{\epsilon}, \psi_{\epsilon}\right)\right|_{\epsilon=0}<0$ (recall the definition of $\left(\theta_{\epsilon}, \phi_{\epsilon}, \psi_{\epsilon}\right)$ from (31)). There are two possible options: (i) we can either construct $\left(\theta_{1}, \phi_{1}, \psi_{1}\right)$ so as to minimize the integrals $\int_{0}^{1}(B-2 \pi M C)\left(\frac{d \phi_{1}}{d s}\right)^{2}+F L^{2} \phi_{1}^{2} d s$ and $\int_{0}^{1} B\left(\frac{d \theta_{1}}{d s}\right)^{2}+\left(F L^{2}-2 \pi M C\right) \theta_{1}^{2} d s$ or (ii) construct $\left(\theta_{1}, \phi_{1}, \psi_{1}\right)$ so as to minimize the integral $\int_{0}^{1} 2 \pi M C\left(\frac{d \phi_{1}}{d s}-\theta_{1}\right)^{2}+C\left(\frac{d \psi_{1}}{d s}\right)^{2} d s$. In what follows, we construct perturbations $\left(\theta_{1}, \phi_{1}, \psi_{1}\right)$ which satisfy (i) and (ii) separately, compare the two different resulting instability criteria and choose the better one.

(i) We set

$$
\begin{aligned}
& \phi_{1}(s)=\sin (\pi s) \\
& \theta_{1}(s)=\sin (\pi s) \\
& \psi_{1}(s)=0 \quad 0 \leq s \leq 1 .
\end{aligned}
$$

We note that $\theta_{1}(0)=\theta_{1}(1)=0$ and $\phi_{1}(0)=\phi_{1}(1)=0$ as required from the clamped boundary condition (4). Substituting (51) into (49), we have that

$$
\left.\frac{d^{2}}{d \epsilon^{2}} \mathcal{E}_{D}\left(\theta_{\epsilon}, \phi_{\epsilon}, \psi_{\epsilon}\right)\right|_{\epsilon=0} \leq 2\left(B \pi^{2}+F L^{2}\right) \int_{0}^{1} \sin ^{2}(\pi s) d s
$$

where we have used the fact that $\int_{0}^{1}\left(\frac{d \sin \pi s}{d s}\right)^{2} d s=\pi^{2} \int_{0}^{1} \sin ^{2}(\pi s) d s$. It is clear that

$$
\left.\frac{d^{2}}{d \epsilon^{2}} \mathcal{E}_{D}\left(\theta_{\epsilon}, \phi_{\epsilon}, \psi_{\epsilon}\right)\right|_{\epsilon=0}<0
$$

if

$$
B \pi^{2}+F L^{2}<0
$$

(ii) We construct $\phi_{1}$ and $\theta_{1}$ so that $\theta_{1}=\frac{d \phi_{1}}{d s}$, subject to the clamped boundary condition (4). There are multiple choices but we set

$$
\begin{aligned}
& \theta_{1}(s)=\sin (2 \pi s), \\
& \phi_{1}(s)=\frac{1}{2 \pi}(1-\cos (2 \pi s)), \\
& \psi_{1}(s)=0 \quad 0 \leq s \leq 1 .
\end{aligned}
$$

We note that the functions $\theta_{1}$ and $\phi_{1}$ vanish at the end-points as required. Substituting (54) into the second variation formula (49), we find that

$$
\left.\frac{d^{2}}{d \epsilon^{2}} \mathcal{E}_{D}\left(\theta_{\epsilon}, \phi_{\epsilon}, \psi_{\epsilon}\right)\right|_{\epsilon=0} \leq F\left(1+\frac{3}{4 \pi^{2}}\right)+4 B \frac{\pi^{2}}{L^{2}}+\frac{B}{L^{2}}-4 \pi M \frac{C}{L^{2}}
$$

and the second variation is negative if

$$
F<\frac{-4 B \frac{\pi^{2}}{L^{2}}-\frac{B}{L^{2}}+4 \pi M \frac{C}{L^{2}}}{\left(1+\frac{3}{4 \pi^{2}}\right)}
$$

We compare formulae (53) and (55); (53) is the classical buckling result with bending stiffness $B$ [25] and (55) depends on the controlled end-rotation characterized by $M$. One might expect (55) to contain more information when $M$ is large. However, we want to compare instability results with the stability result in Proposition 2 . Hence, we work with $B \geq A \geq 2 \pi M C$. Then,

$$
\frac{-4 B \frac{\pi^{2}}{L^{2}}-\frac{B}{L^{2}}+4 \pi M \frac{C}{L^{2}}}{\left(1+\frac{3}{4 \pi^{2}}\right)}<-B \frac{\pi^{2}}{L^{2}}
$$

since $2 B \pi^{2} \geq 4 \pi M C$ and $1+\frac{3}{4 \pi^{2}}<2$. We conclude that under the hypothesis $A \geq 2 \pi M C,(53)$ is a sharper result than (55) and we deduce that the unstressed state defined in (7) and (13) is unstable if

$$
F<-B \frac{\pi^{2}}{L^{2}}
$$


To summarize for the anisotropic case $A \neq B$ with three-dimensional perturbations, we have demonstrated stability of the unstressed state under the condition

$$
\min \left\{A-2 \pi M C, A \frac{\pi^{2}}{L^{2}}+F-2 \pi M \frac{C}{L^{2}},(A-2 \pi M C) \frac{\pi^{2}}{L^{2}}+F\right\}>0
$$

and instability for

$$
F<-B \frac{\pi^{2}}{L^{2}}
$$

The two conditions are compatible since (56) guarantees stability for $A \geq 2 \pi M C$ and

$$
F>F_{c_{1}}=\max \left\{-A \frac{\pi^{2}}{L^{2}}+2 \pi M \frac{C}{L^{2}},-(A-2 \pi M C) \frac{\pi^{2}}{L^{2}}\right\}
$$

and (57) guarantees instability for $F<F_{c_{2}}=-B \frac{\pi^{2}}{L^{2}}$. The two results are consistent since $F_{c_{2}}<F_{c_{1}}$ from (16). The instability condition is analogous to the classical buckling result [25] and does not depend on the twist variable $M$. The stability result does depend on $M$ and one can view $F_{c_{1}}$ as the sum of the classical buckling critical force, $-A \frac{\pi^{2}}{L^{2}}$, with bending stiffness $A$, and a perturbation term, $\left\{2 \pi M \frac{C}{L^{2}}, 2 \pi M C \frac{\pi^{2}}{L^{2}}\right\}$, that depends on the torsional rigidity and the total twist. The stability result is affected by three-dimensional effects whereas the instability result can be captured by in-plane perturbations alone.

We note that the stability result in (2) may not be optimal. This is because the coupling term involving $\phi_{1}^{\prime} \theta_{1}$, in (44), cannot be readily optimally estimated and is indeed the source of the mismatch between the stability and instability criteria. Should the condition $A \geq 2 \pi M C$ be relaxed, then the stability criterion in Proposition 2 does not hold. However, Proposition 3 holds for all $A, M, C>0$ and we have demonstrated that the unstressed state defined in (7) and (13) is unstable if

$$
F<\min \left\{-\frac{B \pi^{2}}{L^{2}}, \frac{-4 B \frac{\pi^{2}}{L^{2}}-\frac{B}{L^{2}}+4 \pi M \frac{C}{L^{2}}}{\left(1+\frac{3}{4 \pi^{2}}\right)}\right\} .
$$

If $B \leq 2 \pi M C$, then it is plausible that the second term in (59) yields a sharper upper bound for the critical force than the classical buckling result.

\subsection{The Isotropic Case}

For an elastic rod with isotropic cross-section, the bending rigidities are equal i.e. $A=B$ in (15) and the rod-energy reduces to the simpler form

$$
\mathcal{E}_{I}[\theta, \phi, \psi]=\int_{0}^{1} \frac{A}{2}\left[\left(\frac{d \phi}{d s}\right)^{2} \sin ^{2} \theta(s)+\left(\frac{d \theta}{d s}\right)^{2}\right]+\frac{C}{2}\left(\frac{d \phi}{d s} \cos \theta(s)+\frac{d \psi}{d s}\right)^{2}-F L^{2} \sin \theta(s) \cos \phi(s) d s
$$

subject to the boundary conditions $\theta(0)=\theta(1)=\frac{\pi}{2}, \phi(0)=\phi(1)=0$ and $\psi(0)=0, \psi(1)=2 \pi M$. The corresponding equilibria are solutions of the associated Euler-Lagrange equations :-

$$
\begin{aligned}
& \phi^{\prime} \cos \theta+\psi^{\prime}=u_{3}=\text { constant } \\
& \frac{d}{d s}\left[A \sin ^{2} \theta+C u_{3} \cos \theta\right]=F L^{2} \sin \theta \sin \phi \\
& A \frac{d^{2} \theta}{d s^{2}}=A \sin \theta \cos \theta\left(\frac{d \phi_{1}}{d s}\right)^{2}-F L^{2} \cos \theta \cos \phi-C u_{3} \sin \theta \frac{d \phi}{d s} .
\end{aligned}
$$

We note that unlike the anisotropic case, all equilibria have constant twist density $u_{3}$.

The unstressed state defined in (7) and (13) is an equilibrium state for the isotropic rod-energy in (60) i.e. is a solution of the Euler-Lagrange equations in (61). We define $F_{c}$ be the critical force such that the unstressed state is a stable equilibrium for $F \geq F_{c}$ and is unstable for $F<F_{c}$. We can obtain explicit upper and lower bounds for $F_{c}$ from Propositions 2 and 3 , by simply setting $A=B$. This yields the following estimates for $F_{c}$ under the hypothesis $A \geq 2 \pi M C$ as shown below:

$$
-A \frac{\pi^{2}}{L^{2}}<F_{c}<\max \left\{-A \frac{\pi^{2}}{L^{2}}+2 \pi M \frac{C}{L^{2}},-(A-2 \pi M C) \frac{\pi^{2}}{L^{2}}\right\} .
$$

In the special case of in-plane perturbations, we recall Proposition 1 and deduce that the unstressed state is stable with respect to in-plane perturbations if $F \geq-A \frac{\pi^{2}}{L^{2}}$ and unstable otherwise. 


\section{Conclusion}

We study the stability of a naturally straight Kirchhoff rod, aligned along the $x$-axis, subject to clamped boundary conditions, controlled end-rotation and terminal loads. We start with a general rod-energy in (6) and show that the diagonal rod-energy in (15) is the most general rod-energy compatible with any planar equilibrium state. We approach the stability analysis from a variational perspective and study the second variation of the diagonal rod-energy, including three-dimensional effects into the problem formulation. The second variation methodology is a more powerful technique than static bifurcation methods and yields rigorous results on the stability spectrum.

By means of a direct computation, we demonstrate that the energy density in (15) is strictly convex with respect to the first derivatives of the Euler angles provided we do not encounter the polar singularities, $\theta=0$ or $\theta=\pi$. The strict convexity is a pre-requisite for the application of Weierstrass theory for scalar variational problems [3,19] to our model problem. We use the positivity of the second variation of the rod-energy as a sufficient criterion for the local energy minimality of the unstressed naturally straight state. From Proposition 2, the naturally straight state is stable for external forces

$$
F>\max \left\{-A \frac{\pi^{2}}{L^{2}}+2 \pi M \frac{C}{L^{2}},-(A-2 \pi M C) \frac{\pi^{2}}{L^{2}}\right\}
$$

provided $A \geq 2 \pi M C$. More precisely, we show that in the parameter regime (58), there exists an $\epsilon$-neighbourhood, $\mathcal{A}_{\epsilon}$, of the unstressed state $\Theta_{0}$ in (7) and (13) for some suitably defined $\epsilon>0$ i.e.

$$
\mathcal{A}_{\epsilon}=\left\{(\theta, \phi, \psi) \in \mathbb{R}^{3} ; \max \left\{\left|\theta(s)-\frac{\pi}{2}\right|,|\phi(s)|,|\psi(s)-2 \pi M s|\right\} \leq \epsilon\right\}
$$

such that

$$
\mathcal{E}_{D}\left(\Theta_{0}\right)<\mathcal{E}_{D}(\theta, \phi, \psi) \quad \forall(\theta, \phi, \psi) \in \mathcal{A}_{\epsilon} .
$$

This conclusion follows directly from the positivity of the second variation of the rod-energy and is the definition of a strict local energy minimum [3,19]. This stability result is accompanied by an instability result in Proposition 3 where we demonstrate instability of the naturally straight state for $F<-B \frac{\pi^{2}}{L^{2}}$. Therefore, if $F_{c}$ denotes the critical force such that the naturally straight state is stable for $F \geq F_{c}$ and unstable for $F<F_{c}$, then

$$
-B \frac{\pi^{2}}{L^{2}}<F_{c}<\max \left\{-A \frac{\pi^{2}}{L^{2}}+2 \pi M \frac{C}{L^{2}},-(A-2 \pi M C) \frac{\pi^{2}}{L^{2}}\right\}
$$

where $A \leq B$. The two-dimensional in-plane analysis is simpler whereby we recover the classical buckling result in Proposition 1 .

The results in this paper are an example of how variational methods can be applied to stability problems in the theory of Kirchhoff rods, yielding rigorous results in a three-dimensional framework. Our methods highlight the mathematical analogies between the anisotropic case $(A \neq B)$ and the isotropic case $(A=B)$, see Propositions 2 and 3 where we effectively reduce the anisotropic problem to the isotropic one. We use integral inequalities to obtain bounds for the second variation and this is an approach that can be further exploited. We hope to generalize these techniques to a wide class of problems in elasticity with special emphasis on the structure and stability of buckled modes, subject to topological constraints e.g. fixed linking number [13] in future work.

\section{Acknowledgements}

This publication is based on work supported by Award No. KUK-C1-013-04, made by King Abdullah University of Science and Technology (KAUST). AG is a Wolfson Royal Society Merit Holder. AM is also supported by an EPSRC Career Acceleration Fellowship EP/J001686/1. We also would like to thank John Maddocks for comments on an earlier version of this manuscript, and bringing to our attention important references.

\section{References}

1. Antman S. S., Kenney C. S.: Large buckled states of nonlinearly elastic rods under torsion, thrust and gravity. Arch. Ration Mech. Anal. 84, 289-338 (1981).

2. Antman S. S.: Nonlinear Problems in Elasticity. Springer, New York (1995).

3. Ball, J., Marsden, J.E.: Quasiconvexity at the boundary, positivity of the second variation, and elastic stability. Arch. Rat. Mech. Anal., 86, 251-277, (1984).

4. Bazant Z. P., Cedolin L.: Stability of structures: elastic, inelastic, fracture, and damage theories. Dover Publications, (2003). 
5. Beck M.: Die knicklast des einseitig eigenspanntan tangential gedrückten. Zeitschrift für angewandte Mathematik und Mechanik, (1952).

6. Browne R.C.: Dynamic stability of one-dimensional nonlinearly viscoelastic bodies. Arch. Ration. Mech. Anal. 68(3), 257-282 (1978).

7. Born M.: Untersuchungen über die stabilität der elastischen Linie in Ebene und Raum: Unter verschiedenen Grenzbedingungen. Dieterich, Göttingen, (1906).

8. Chouaieb N., Goriely A., Maddocks J. H.: Helices. Proc. Natl. Acad. Sci. USA. 103, 9398-9403 (2006).

9. Chouaieb N, Maddocks J.: Kirchhoffs Problem of Helical Equilibria of Uniform Rods. J. Elast. 77, 221-247 (2004).

10. Euler. L: Methodus inveniendi lineas curvas maximi minimive proprietate gaudentes sive solutio problematis isoperimetrici latissimo sensu accepti. apud Marcum-Michaelem Bousquet \& socios, (1744).

11. Euler L.: Sur la force des colonnes. Mem. Acad. Berlin 13, (1759).

12. Evans L.: Partial Differential Equations. American Mathematical Society, Providence (1998).

13. Fain B., Rudnick J., Ostlund S.: Conformations of linear DNA. Phys. Rev. E. 55(6), 7364-7367 (1997).

14. Gore J., Bryant Z., Nöllmann M., Le M.U., Cozzarelli N.R., Bustamante C.: DNA overwinds when stretched. Nature. 442(7104), 836-839 (2006).

15. Goriely A.: Twisted elastic rings and the rediscoveries of Michell's instability. J. Elast. 84(3), 281-299 (2006)

16. Goriely A., Nizette M., Tabor M.: On the Dynamics of Elastic Strips. J. Nonlinear Sci. 11(1), 3-45 (2001).

17. Goriely A., Tabor M.: Nonlinear dynamics of filaments I: Dynamical instabilities. Physica D. 105, 20-44 (1997).

18. Greenhill A. G.: On the strength of shafting when exposed both to torsion and to end thrust. ARCHIVE: Proceedings of the Institution of Mechanical Engineers 1847-1982 (vols 1-196). 34(1883), 182-225, 1883.

19. Hestenes, M.R.: Calculus of variations and optimal control theory. Wiley (1966).

20. Love A. E. H.: A treatise on the Mathematical Theory of Elasticity. 4th edition Dover, New York (1944).

21. Maddocks J. H.: Stability of nonlinearly elastic rods. Arch. Ration. Mech. Anal. 85(4), 180-198 (1984).

22. Manning R.S., Rogers K.A., Maddocks J.H.: Isoperimetric conjugate points with application to the stability of dna minicircles. Proc. Royal Soc. London. A: Math. Phys. Eng. Sci., 454, 3047- (1998).

23. Michell. J. H.: On the stability of a bent and twisted wire. Messag. Math., 11, (1889)

24. Nizette M., Goriely A.: Toward a classification of Euler-Kirchhoff filaments. J. Math. Phys. 40, 2830-2866 (1999).

25. O'Reily O. M., Peters D. M.: On Stability Analyses of Three Classical Buckling Problems for the Elastic Strut. J. Elast. 105, 117-136 (2011)

26. Timoshenko S. P., Gere J. M.: Theory of elastic stability. Mcgraw-Hill, New York (1961).

27. Virga E. G.: An extended Wirtinger inequality. J. Phys. A: Math. Gen. 34, 1507-1511 (2001).

28. Ziegler. H.: Principles of structural stability.: Waltham, Mass:Blaisdell (1968). 



\section{RECENT REPORTS}

41/11 On the modelling and simulation of a high pressure shift freezing Smith process

Peppin

Ángel M. Ramos

42/11 An efficient implementation of an implicit FEM scheme for fractional-in-space reaction-diffusion equations

Burrage

Hale

Kay

43/11 Coupling fluid and solute dynamics within the ocular surface tear film: a modelling study of black Line osmolarity

Zubkov

Breward

Gaffney

44/11 A prototypical model for tensional wrinkling in thin sheets

Davidovitch

Schroll

Vella

Adda-Bedia

Cerda

45/11 A fibrocontractive mechanochemical model of dermal wound closure incorporating realistic growth factor

Murphy

Hall

Maini

McCue

McElwain

46/11 A two-compartment mechanochemical model of the roles of transforming growth factor $\beta$ and tissue tension in dermal wound healing

Murphy

Hall

Maini

$\mathrm{McCue}$

McElwain

47/11 Effects of demographic noise on the synchronization of a metapopulation in a fluctuating environment

Lai

Newby

Bressloff

48/11 High order weak methods for stochastic differential equations based on modified equations

Abdulle

Cohen

Vilmart

Zygalakis

49/11 The kinetics of ice-lens growth in porous media

Style

Peppin

50/11 Wound healing angiogenesis: the clinical implications of a simple

Flegg mathematical model

Byrne

Flegg

McElwain

51/11 Wound healing angiogenesis: the clinical implications of a simple mathematical model

$\mathrm{Du}$

Gunzburger

Lehoucq

Zhou

52/11 Image Inpainting based on coherence transport with Adapted dis-

März tance functions

53/11 Surface growth kinematics via local curve evolution

Moulton

Goriely

54/11 A multiple scales approach to evaporation induced Marangoni Hennessey 
55/11 The dynamics of bistable liquid crystal wells

Luo

Majumdar

Erban

56/11 Real-Time Fluid Effects on Surfaces using the Closest Point Method

Auer

Macdonald

Treib

Schneider

Westermann

$57 / 11$ Isolating intrinsic noise sources in a stochastic genetic switch

Newby

58/11 Riemann-Cartan Geometry of Nonlinear Dislocation Mechanics

Yavari

Goriely

59/11 Helices through 3 or 4 points?

Goriely

Neukirch

Hausrath

60/11 Bayesian data assimilation in shape registration

Cotter

Cotter

Vialard

61/11 Asymptotic solution of a model for bilayer organic diodes and solar cells

Richardson

Please

Kirkpatrick

62/11 Neural field model of binocular rivalry waves

Bressloff

Webber

63/11 Front propagation in stochastic neural fields

Bressloff

Webber

Copies of these, and any other OCCAM reports can be obtained from:

Oxford Centre for Collaborative Applied Mathematics Mathematical Institute

24 - 29 St Giles'

Oxford

OX1 3LB

England

www.maths.ox.ac.uk/occam 